\title{
Venous and arterial thrombosis:Two aspects of the same disease?
}

\section{Paolo Prandoni}

Department of Cardiothoracic and Vascular Sciences, Thromboembolism Unit, University Hospital of Padua Padua, Italy
Correspondence: Paolo Prandoni Department of Cardiothoracic and Vascular Sciences, Thromboembolism Unit, University of Padua, Via Giustiniani 2, 35128 - Padua, Italy

$\mathrm{Tel}+390498212656$

Fax +39049821378I

Email paoloprandoni@tin.it

\begin{abstract}
An increasing body of evidence suggests the likelihood of a link between venous and arterial thrombosis. The two vascular complications share several risk factors, such as age, obesity, diabetes mellitus, blood hypertension, hypertriglyceridemia, and metabolic syndrome. Moreover, there are many examples of conditions accounting for both venous and arterial thrombosis, such as the antiphospholipid antibody syndrome, hyperhomocysteinemia, malignancies, infections, and the use of hormonal treatment. Finally, several recent studies have consistently shown that patients with venous thromboembolism are at a higher risk of arterial thrombotic complications than matched control individuals. We, therefore, speculate the two vascular complications are simultaneously triggered by biological stimuli responsible for activating coagulation and inflammatory pathways in both the arterial and the venous system. Future studies are needed to clarify the nature of this association, to assess its extent, and to evaluate its implications for clinical practice.
\end{abstract}

Keywords: venous thromboembolism, deep vein thrombosis, pulmonary embolism, myocardial infarction, ischemic stroke, atherosclerosis

\section{Introduction}

Venous and arterial thrombotic disorders have long been viewed as separate pathophysiological entities, partly as a result of the obvious anatomical differences, as well as their distinct clinical presentations. In particular, arterial thrombosis has long been held to be largely a phenomenon of platelet activation, whereas venous thrombosis is largely a matter of activation of the clotting system. However, there is evidence that this dichotomy is likely to be an oversimplification. Fibrin-rich thrombi form in the left atrial appendage of patients with atrial fibrillation and in the coronary artery system of patients with myocardial infarction. Accordingly, anticoagulant drugs are highly effective for prevention of arterial embolism related to atrial fibrillation, ${ }^{1}$ and for prevention and treatment of coronary artery disease. ${ }^{2}$ Likewise, platelets play an inevitable role in the formation of thrombi in the venous system, and antiplatelet agents have been shown to be effective for prevention of venous thromboembolic disorders, although to a smaller extent than anticoagulant drugs. ${ }^{3,4}$ As another example, subjects who sustain a retinal vein thrombosis commonly have associated cardiovascular risk factors, ${ }^{5}$ and the causes of mortality on follow-up are usually arterial vascular events. ${ }^{6}$

\section{Association between venous thromboembolism (VTE) and atherosclerosis}

A potential association between venous thromboembolism (VTE) and atherosclerosis was described in 2003. ${ }^{7}$ Ultrasonography of the carotid arteries was performed in 299 unselected patients with deep vein thrombosis (DVT) without symptomatic atherosclerosis and in 150 control subjects. In a multivariate analysis taking into account risk factors for atherosclerosis, the odds ratio (OR) for carotid plaques in patients with 
unprovoked as compared to secondary DVT and controls was found to be 2.4, and was highly statistically significant.

Subsequently, two studies provided further evidence supporting the association between VTE and atherosclerosis. In a case-control study, Hong and colleagues found a higher prevalence of coronary artery calcium, as assessed by chest computed tomography (CT) scan, in patients with unprovoked VTE than in matched control individuals. ${ }^{8}$ In a series of almost 24,000 consecutive autopsies, Eliasson and colleagues found an increased prevalence of VTE in patients with arterial thrombosis, except for those with coronary artery thrombosis. ${ }^{9}$

Recently, Ageno and colleagues reviewed the evidence favoring the association of the most important risk factors for atherosclerosis and VTE. ${ }^{10}$ After reviewing 21 case-control and cohort studies dealing with a total of 63,552 patients meeting the inclusion criteria, factors that were found to be significantly associated with an increased risk of VTE were obesity, blood hypertension, diabetes mellitus, and hypertriglyceridemia. Of interest, weighted mean high-density lipoprotein cholesterol levels were significantly lower in VTE patients, whereas no difference between VTE and non-VTE patients for total and low-density lipoprotein cholesterol levels was found. ${ }^{10}$

Although information coming from the above reported investigations suggests the existence of an association between VTE and atherosclerosis, it does not clarify the nature of this association. On the one hand, atherosclerosis has the potential to promote the development of thrombotic disorders in the venous system. Atherosclerosis is associated with a detectable activation of both platelets and blood coagulation as well as an increased fibrin turnover, which can lead to thrombotic complications. ${ }^{11-14}$ The role of this prothrombotic state in favoring venous thrombotic events is plausible given the assumption that activated platelets and coagulation factors appear in the slow-flowing venous system. On the other hand, the two clinical conditions may share common mechanisms or risk factors. In nature, there are many examples of conditions accounting for both arterial and venous thromboembolic disorders, such as hyperhomocysteinemia, ${ }^{15}$ antiphospholipid antibodies, ${ }^{16}$ malignancies, ${ }^{17}$ paroxysmal nocturnal hemoglobinuria, ${ }^{18}$ infectious states, ${ }^{19}$ and the use of hormonal therapy. ${ }^{20}$

\section{Is atherosclerosis predictive of VTE?}

In an attempt to assess whether atherosclerotic disease predisposes to VTE, the authors of two similar populationbased cohort studies carried out in the US, the Atherosclerosis
Risk in Communities and the Cardiovascular Health Study, evaluated the rate of VTE development in subjects younger and older than 65, respectively, who had carotid ultrasound and the assessment of other subclinical parameters of atherosclerosis, and were then followed-up prospectively for several years. ${ }^{21,22}$

In the former study, 13,081 adults aged 45-64 years underwent carotid ultrasonography to assess the intimamedia thickness and the presence of atherosclerotic plaques. ${ }^{21}$ After adjustment for age, sex, ethnicity, body mass index, and diabetes, no association was found between ultrasound parameters of subclinical atherosclerosis and VTE development after a mean follow-up of 12.5 years (adjusted hazard ratio [HR] of VTE for presence of carotid plaques, $0.97 ; 95 \%$ confidence interval [CI], 0.72 to 1.29 ).

In the latter study, 4108 individuals aged at least 65 years underwent non-invasive assessment of subclinical atherosclerosis using carotid ultrasound (intima-media thickness and presence of plaques), ankle-brachial blood pressure index and electrocardiogram, and then were followed-up for a median of 11.7 years. $^{22}$ Surprisingly enough, the adjusted relative risk (RR) of overall and idiopathic VTE for presence of any type of subclinical atherosclerosis was 0.60 (95\% CI, 0.39 to 0.91$)$ and 0.32 (95\% CI, 0.18 to 0.59$)$, respectively. These unexpected findings were mostly explained by an inverse association of high-risk carotid plaques or arterial events during follow-up.

Based on these findings, asymptomatic atherosclerosis is unlikely to constitute a risk factor of venous thromboembolic disorders. Whether patients developing symptomatic complications of atherosclerosis such as myocardial infarction or stroke are at a higher risk of VTE complications is controversial. While in the former of the two above reported studies, the occurrence of cardiovascular and cerebrovascular events was significantly associated with the development of VTE, ${ }^{21}$ in the latter the opposite was seen. ${ }^{22}$ In an attempt to determine the impact of cardiovascular risk factors, including family history of myocardial infarction, on the incidence of VTE, Braekkan and colleagues extracted data from 21,330 subjects, aged 25-96 years, who had been enrolled in a prospective, population-based study (the Tromsø Study). ${ }^{23}$ In multivariable analysis, family history of myocardial infarction was significantly associated with both total VTE (HR, 1.27; 95\% CI, 1.01-1.60) and unprovoked VTE (HR, 1.46; 95\% CI, 1.03-2.07. Finally, in a recent population-based study conducted in Denmark patients with a history of arterial cardiovascular events had a clearly increased relative risk of VTE events in the first three 
months following the index event, then the risk decreased yet remained statistically significant. ${ }^{24}$

\section{Is VTE predictive of arterial cardiovascular events?}

Another scenario assumes that the same biological trigger is responsible for activating coagulation and inflammatory pathways in both arterial and venous thromboembolism, in such a way determining a simultaneous risk of arterial and venous thrombotic complications. If this is true, subjects with VTE may be at a higher risk of subsequent arterial cardiovascular events than matched control individuals. This hypothesis has recently been tested in seven studies (summarized in Table 1).

The incidence of arterial cardiovascular events after VTE was assessed for the first time in a long-term prospective study on the long-term follow-up in 360 patients with a first episode of pulmonary embolism (PE), of whom 209 with idiopathic PE and 151 with $\mathrm{PE}$ associated with transient risk factors. ${ }^{25}$ The study outcomes were cardiovascular events (recurrent VTE, acute myocardial infarction, stroke, sudden otherwise unexplained death), cardiovascular death, and death due to any cause. After a median follow-up of 38 months, a cardiovascular event occurred in 47 patients with idiopathic PE (7.5\% patient-year, and in 17 patients with PE associated with transient risk factors (3.1\% patient-year) (RR, 2.0; 95\% CI, 1.20 to 3.34$)$. Twenty patients with idiopathic PE (3.2\% patient-year) and 2 patients with $\mathrm{PE}$ associated with transient risk factors $(0.4 \%$ patient-year) presented with an arterial cardiovascular events (RR, 7.2; 95\% CI, 1.71 to 30.45). After adjusting for age, the idiopathic nature of the index PE was confirmed to be an independent risk factor for arterial cardiovascular events at the long-term follow-up.

Later, we reported the results of a prospective follow-up study in 1919 consecutive patients with a first VTE episode. ${ }^{26}$ The primary outcome was nonfatal and fatal symptomatic atherosclerotic disease (including fatal and nonfatal coronary heart disease, fatal and nonfatal ischemic stroke, symptomatic peripheral artery disease, fatal heart failure from ischemic and/or hypertensive cardiopathy, and sudden otherwise inexplicable death) in patients with VTE of unknown origin as compared to those with secondary VTE. After a median follow-up of 48 and 51 months, respectively, at least one symptomatic atherosclerotic complication was detected in 160 of the 1063 patients (15.1\%) with VTE of unknown origin, and in 73 of the $856(8.5 \%)$ with secondary VTE. After adjusting for age and other risk factors of atherosclerosis, the HR for symptomatic atherosclerotic complications in patients with VTE of unknown origin compared to those with secondary VTE was $1.6(95 \%$ CI, 1.2-2.0). When the analysis was restricted to patients without previous symptomatic atherosclerosis, the HR became 1.7 (95\% CI, 1.1-2.4).

In the recently published study by Schulman and colleagues, reporting on the extended 10-year follow-up of the DURAC study in 897 patients with VTE who had been randomized to receive six weeks or six months of vitamin $\mathrm{K}$ antagonists, ${ }^{27}$ the overall mortality $(28.5 \%)$ was higher than that expected in the general population with a standardized incidence ratio (SIR) of 1.43 (95\% CI, 1.28 to 1.58$)$, mainly because of a higher mortality than expected from cancer (SIR, 1.83; 95\% CI, 1.44 to 2.23 ), and from myocardial infarction or stroke (SIR, 1.28; $95 \% \mathrm{CI}, 1.00$ to 1.56$){ }^{28}$

Table I Main studies addressing the risk of arterial cardiovascular events in patients with VTE

\begin{tabular}{|c|c|c|c|c|}
\hline Ref & Study design & Study population & Number & Findings \\
\hline 25 & Prospective cohort & $\begin{array}{l}\text { Idiopathic PE } \\
\text { Secondary PE }\end{array}$ & $\begin{array}{l}209 \\
151\end{array}$ & $\begin{array}{l}\text { Idiopathic PE risk factor of arterial } \\
\text { cardiovascular events }\end{array}$ \\
\hline 26 & Prospective cohort & $\begin{array}{l}\text { Idiopathic VTE } \\
\text { Secondary VTE }\end{array}$ & $\begin{array}{l}1,063 \\
856\end{array}$ & $\begin{array}{l}\text { Idiopathic VTE risk factor of arterial } \\
\text { cardiovascular events }\end{array}$ \\
\hline 28 & $\begin{array}{l}\text { Extended follow-up } \\
\text { of the DURAC study }{ }^{27}\end{array}$ & $\begin{array}{l}\text { VTE patients } \\
\text { General Swedish population }\end{array}$ & 897 & $\begin{array}{l}\text { Higher mortality from myocardial } \\
\text { infarction or stroke in VTE patients }\end{array}$ \\
\hline 29 & Retrospective cohort & DVT patients Controls & $\begin{array}{l}151 \\
151\end{array}$ & $\begin{array}{l}\text { Idiopathic VTE risk factor of arterial } \\
\text { cardiovascular events }\end{array}$ \\
\hline 30 & Prospective cohort & $\begin{array}{l}\text { DVT with residual thrombosis } \\
\text { DVT with recanalized veins }\end{array}$ & $\begin{array}{l}173 \\
143\end{array}$ & $\begin{array}{l}\text { Residual thrombosis risk factor } \\
\text { of vascular death }\end{array}$ \\
\hline 32 & Population-based & VTE patients Controls & $\begin{array}{l}42,124 \\
163,566\end{array}$ & $\begin{array}{l}\text { VTE risk factor of myocardial infarction } \\
\text { or stroke }\end{array}$ \\
\hline 33 & Population-based & Idiopathic VTE Controls & $\begin{array}{l}6,065 \\
12,040\end{array}$ & $\begin{array}{l}\text { Idiopathic VTE in patients aged }<40 \\
\text { risk factor of myocardial infarction }\end{array}$ \\
\hline
\end{tabular}

Abbreviations: DVT, deep vein thrombosis; PE, pulmonary embolism; VTE, venous thromboembolism. 
Bova and colleagues explored the rate of subsequent arterial events in patients with unprovoked VTE and control subjects. ${ }^{29}$ In a retrospective cohort study, they compared the rate of subsequent arterial events (ie, acute myocardial infarction, ischemic stroke and peripheral arterial disease) in 151 consecutive patients with objectively confirmed spontaneous VTE and 151 control subjects randomly selected from the database of two family physicians. They collected information about cardiovascular risk factors (hypertension, hypercholesterolemia, diabetes, obesity, and smoke) at the time of VTE episode, or corresponding date for the controls, and considered the follow-up from this time. During a mean follow-up of 43 months there were 16 arterial events in the VTE patients and six in the control group, corresponding to an HR of 2.84; $95 \% \mathrm{CI}, 1.11$ to 7.27 . The difference remained significant after adjusting for age and other cardiovascular risk factors (adjusted HR, 2.86; 95\% CI, 1.07 to 7.62 ). Overall mortality was also higher in the VTE patients (12 vs 4 deaths).

Young and colleagues followed over five years a cohort of 316 patients with acute DVT. ${ }^{30}$ The patients were divided into those with completely clear vessels on follow-up scan (45\%) and those with residual thrombus identified (55\%). As expected, patients with residual thrombus on follow-up ultrasound were at higher risk of recurrence, which remained significant after multivariate adjustment for age, gender and malignancy (adjusted HR, 2.2; 95\% CI, 1.15 to 4.17). During follow-up, the cumulative mortality was $12 \%$ at two years and $27 \%$ by five years. The risk of death was increased in patients with residual thrombus at follow-up scan, and this risk persisted after multivariate analysis of age, gender and malignancy (adjusted RR, 2.8; 95\% CI, 1.37 to 5.72). Although the majority of deaths were due to malignancy (68\%), 10 patients (18\%) died from vascular causes. There was a trend towards increased vascular death in the patients with residual thrombus on follow up ultrasound scan, which did not reach significance (HR, 4.1; 95\% CI, 0.87 to 19.33 ). The increased risk of death in patients with residual thrombus, with a trend towards increased vascular death, may suggest that failure of thrombus resolution, a well known risk factor of recurrent thromboembolism, ${ }^{31}$ is a marker of more global vascular dysfunction.

In a recent population-based cohort study using nationwide Danish medical databases, Sorensen and colleagues assessed the risk of hospitalization due to myocardial infarction, stroke and transient ischemic attack among 25,199 patients with DVT, 16,925 patients with PE, and 163,566 population controls. ${ }^{32}$ Patients with both
DVT and PE were found to have a substantially increased risk of myocardial infarction and stroke during the first year after the thrombotic event. For patients with DVT, the relative risks varied from 1.60 for myocardial infarction (95\% CI, 1.35-1.91) to 2.19 (95\% CI, 1.85-2.60) for stroke. For patients with PE, the relative risks were 2.60 (95\% CI, 2.14-3.14) for myocardial infarction and 2.93 (95\% CI, 2.34-3.66) for stroke. The relative risks were also elevated, though less markedly, during the subsequent 20 years of follow-up, with $20 \%$ to $40 \%$ increases in risk for arterial cardiovascular events. Relative risks were similar for those with provoked and unprovoked DVT and PE.

Spencer and colleagues performed a longitudinal matched cohort study utilizing multiple administrative databases. ${ }^{33}$ Six thousand and sixty five Ontario residents aged 20-64 years diagnosed with unprovoked VTE from 1 April 1991 to 31 March 1995 were matched to a population cohort $(n=12,040)$ in 1:2 fashion on the basis of age, gender, socioeconomic class, cardiovascular risk factors and other comorbidities. Patients 20-39 years of age presenting with unprovoked VTE had an increased risk of myocardial infarction (HR, 3.92; 95\% CI, 1.65-9.35) as compared to controls; the association was applicable to those without atherosclerotic risk factors at baseline. There was no significant relationship between unprovoked VTE and AMI among patients 40-64 years old, with or without atherosclerotic risk factors. Irrespective of age, patients with unprovoked VTE had an increased risk of all-cause death or our composite endpoint of AMI and/or death as compared to patients without VTE.

All together, the results of these studies are in keeping with the observation of a higher prevalence of carotid atherosclerosis in patients with unprovoked DVT than in matched control individuals, ${ }^{7}$ and suggest that patients with VTE have also an increased risk of subsequent symptomatic arterial cardiovascular events. This implies that arterial and venous thrombosis may share common mechanisms or risk factors, and may have a common origin in abnormalities of various blood constituents. We speculate that venous and arterial thrombosis are two aspects of the same disease (ie, thrombosis), and that this disease may electively affect genetically predisposed individuals resulting in clinically manifestations that are, in turn, depending on a variety of elements including the age of patients, their lifestyle, and the occurrence of co-morbidities and circumstantial factors: the venous thrombotic events being more frequent, for example, after triggering risk factors such as surgery or trauma, and the arterial thrombotic events being more frequent in subjects who have developed atherosclerosis. 


\section{Conclusions and implications of the association}

These findings have several implications for both research and medical practice. Patients with VTE of unknown origin could be examined for asymptomatic atherosclerosis, in order to modify aggressively the risk profile in those with abnormal test results. Measures could include appropriate counseling about lifestyle changes and control of risk factors for atherosclerosis. Interestingly enough, lifestyle factors are likely to have a major impact on the risk of VTE. A diet including more plant food and fish and less red and processed meat,${ }^{34}$ the supplementation of vitamin $\mathrm{E},{ }^{35}$ regular sports activities, ${ }^{36}$ and alcohol consumption ${ }^{37}$ have recently been found to be associated with a lower incidence of venous thrombosis. Consistent with these findings are the results of a prospective cohort study conducted in Sweden on 40,000 Swedish women who were followed-up for a mean of 11 years: female nonsmokers who were physically active and who consumed alcohol in moderation were found to have a lower risk of VTE. ${ }^{38}$

In addition, a potential role for prophylaxis of both recurrent VTE and arterial cardiovascular events with antiplatelet therapy or statins may be explored. Interest in statins has increased, given recent data that suggest a potential role both in controlling the development of atherosclerotic lesions and in lowering the risk of venous thromboembolism. ${ }^{39}$

The currently available evidence for a role of aspirin in VTE prevention and treatment is quite limited. Data on the long-term management of VTE will be provided by two twin studies currently ongoing in Italy (Warfasa) and in Australia and New Zealand (Aspire). ${ }^{40}$ Both studies are aimed at assessing the clinical benefit of $100 \mathrm{mg}$ of aspirin given after the completion of anticoagulant treatment in patients with a first episode of unprovoked VTE.

In conclusion, the separate nature of arterial and venous thrombotic disorders has been challenged. Future studies are needed to clarify the nature of this association, to assess its extent, and to evaluate its implications for clinical practice.

\section{Disclosure}

The authors report no conflicts of interest in this work.

\section{References}

1. Singer DE, Albers GW, Dalen JE, et al. Antithrombotic therapy in atrial Fibrillation. American College of Chest Physicians evidence-based clinical practice guidelines (8th edition). Chest. 2008;133(6 Suppl):546S-592S.

2. Becker RC, Meade TW, Berger PB, et al. The primary and secondary prevention of coronary artery disease. American College of Chest Physicians evidence-based clinical practice guidelines (8th edition). Chest. 2008;133(6 Suppl):776S-814S.
3. Antithrombotic Trialist's Collaboration. Collaborative meta-analysis of randomised trials of antiplatelet therapy for prevention of death, myocardial infarction, and stroke in high-risk patients. $\mathrm{Br} \mathrm{Med} J$. 2002;324(7329):71-86.

4. Hovens MM, Snoep JD, Tamsma JT, Huisman MV. Aspirin in the prevention and treatment of venous thromboembolism. J Thromb Haemost. 2006;4(7):1470-1475.

5. Janssen MC, den Heijer M, Cruysberg JR, Wollersheim H, Bredie SJ. Retinal vein occlusion: a form of venous thrombosis or a complication of atherosclerosis? A meta-analysis of thrombophilic factors. Thromb Haemost. 2005;93(6):1021-1026.

6. Tsaloumas MD, Kirwan J, Vinall H, et al. Nine year follow-up study of morbidity and mortality in retinal vein occlusion. Eye. 2000;14(Pt 6):821-827.

7. Prandoni P, Bilora F, Marchiori A, et al. An association between atherosclerosis and venous thrombosis. $N$ Engl J Med. 2003;348(15): 1435-1441.

8. Hong C, Zhu F, Du D, Pilgram TK, Sicard GA, Bae KT Coronary artery calcification and risk factors for atherosclerosis in patients with venous thromboembolism. Atherosclerosis. 2005;183(1):169-174.

9. Eliasson A, Bergqvist D, Bjorck M, Acosta S, Sternby NH, Ogren M. Prevalence and risk of venous thromboembolism in patients with verified arterial thrombosis: a population study based on 23796 consecutive autopsies. J Thromb Haemost. 2006;4(9):1897-1902.

10. Ageno W, Becattini C, Brighton T, Selby R, Kamphuisen PW. Cardiovascular risk factors and venous thromboembolism: a meta-analysis. Circulation. 2008;117(1):93-102.

11. Meade TW, Mellows S, Brozovic M, Miller GJ, Chakrabarti RR, North WR, Haines AP, Stirling Y, Imeson JD, Thompson SG. Haemostatic function and ischemic heart disease: principal results from the Northwick Park Heart Study. Lancet. 1986;2(8506):533-537.

12. Thompson SG, Kienast J, Pyke SD, Haverkate F, van de Loo JC. Hemostatic factors and the risk of myocardial infarction or sudden death in patients with angina pectoris. European Concerted Action on Thrombosis and Disabilities Angina Pectoris Study Group. $N$ Engl J Med. 1995;332(10):635-641.

13. Folsom AR, Wu KK, Rosamond WR, Sharrett AR, Chambless LE. Prospective study of hemostatic factors and incidence of coronary heart disease: the Atherosclerosis Risk in Communities (ARIC) Study. Circulation. 1997;96(4):1102-1108.

14. Koenig W, Rothenbacher D, Hoffmeister A, Griesshammer M, Brenner H. Plasma fibrin D-dimer levels and risk of stable coronary artery disease: results of a large case-control study. Arterioscler Thromb Vasc Biol. 2001;21(10):1701-1705.

15. Guthikonda S, Haynes WG. Homocysteine: role and implications in atherosclerosis. Curr Atheroscler Rep. 2006;8(2):100-106.

16. Erkan D, Lockshin MD. Antiphospholipid syndrome. Curr Opin Rheumatol. 2006;18(3):242-248.

17. Grisold W, Oberndorfer S, Struhal W. Stroke and cancer: a review. Acta Neurol Scand. 2008; Jun 20 [Epub ahead of Print].

18. Omine M, Kinoshita T, Nakakuma H, Maciejewski JP, Parker CJ, Socie G. Paroxysmal nocturnal hemoglobinuria. Int J Hematol. 2005;82(5):417-421.

19. Levine RL, Leclerc JR, Bailey JE, Monberg MJ, Sarwat S. Venous and arterial thromboembolism in severe sepsis. Thromb Haemost. 2008;99(5):892-898.

20. Sare GM, Gray LJ, Bath PM. Association between hormone replacement therapy and subsequent arterial and venous vascular events: a meta-analysis. Eur Heart J. 2008;29(16):2031-2041.

21. Reich LM, Folsom AR, Key NS, et al. Prospective study of subclinical atherosclerosis as a risk factor for venous thromboembolism. J Thromb Haemost. 2006;4(9):1903-1908.

22. van der Hagen PB, Folsom AR, Jenny NS, et al. Subclinical atherosclerosis and the risk of future venous thrombosis in the Cardiovascular Health Study. J Thromb Haemost. 2006;4:4(9): 1903-1908. 
23. Brækkan SK, Mathiesen EB, Njølstad I, Wilsgaard T, Størmer J, Hansen JB. Family history of myocardial infarction is an independent risk factor for venous thromboembolism - the Tromsø study. J Thromb Haemost. 2008;6(11):1851-1857.

24. Sørensen HT, Horvath-Puho E, Kobberøe Søgaard K, et al. Arterial cardiovascular events, statins, low dose aspirin and subsequent risk of venous thromboembolism: a population based case control study. J Thromb Haemost. 2009:In Press.

25. Becattini C, Agnelli G, Prandoni P, et al. A prospective study on cardiovascular events after acute pulmonary embolism. Eur Heart J. 2005;26(1):77-83.

26. Prandoni P, Ghirarduzzi A, Prins MH, et al. Venous thromboembolism and the risk of subsequent symptomatic atherosclerosis. J Thromb Haemost. 2006;4(9):1891-1896.

27. Schulman S, Rhedin AS, Lindmarker P, et al. A comparison of six weeks with six months of oral anticoagulant therapy after a first episode of venous thromboembolism. $N$ Engl J Med. 1995;332(25):1661-1665.

28. Schulman S, Lindmarker P, Holmstrom M, et al. Post-thrombotic syndrome, recurrence, and death 10 years after the first episode of venous thromboembolism treated with warfarin for 6 weeks or 6 months. J Thromb Haemost. 2006;4(4):734-742.

29. Bova C, Marchiori A, Noto A, et al. Incidence of arterial cardiovascular events in patients with idiopathic venous thromboembolism. A retrospective cohort study. Thromb Haemost. 2006;96(2):132-136.

30. Young L, Ockelford P, Milne D, Rolfe-Vyson V, McKelvie S, Harper P. Post treatment residual thrombus increases the risk of recurrent deep vein thrombosis and mortality. J Thromb Haemost. 2006;4(9):1919-1924.

31. Prandoni P, Lensing AWA, Prins MH, et al. Residual venous thrombosis as a predictive factor of recurrent venous thromboembolism. Ann Intern Med. 2002;4(9):1919-1924.
32. Sørensen HT, Horvath-Puho E, Pedersen L, Baron JA, Prandoni P. Venous thromboembolism and subsequent hospitalization due to acute arterial cardiovascular events - A 20 year cohort study. Lancet. 2007;370(9601):1773-1779.

33. Spencer FA, Ginsberg JS, Chong A, Alter DA. The relationship between unprovoked venous thromboembolism, age, and acute myocardial infarction. J Thromb Haemost. 2008;6(9):1507-1513.

34. Steffen LM, Folsom AR, Cushman M, Jacobs DR Jr, Rosamond WD. Greater fish, fruit, and vegetable intakes are related to lower incidence of venous thromboembolism. The Longitudinal Investigation of Thromboembolism Etiology. Circulation. 2006;15(2):188-195.

35. Glynn RJ, Ridker PM, Goldhaber SZ, Zee RY, Buring JE. Effects of random allocation to vitamin e supplementation on the occurrence of venous thromboembolism. Report from the Women's Health Study. Circulation. 2007;116(13):1497-1503.

36. van Stralen KJ, Le Cessie S, Rosendaal FR, Doggen CJ. Regular sports activities decrease the risk of venous thrombosis. $J$ Thromb Haemost. 2007;5(11):2186-2192.

37. Pomp ER, Rosendaal FR, Doggen CJ. Alcohol consumption is associated with a decreased risk of venous thrombosis. Thromb Haemost. 2008;99(1):59-63.

38. Lindqvist PG, Epstein E, Olsson H. The relationship between lifestyle factors and venous thromboembolism among women: a report from the MISS study. Br J Haematol. 2008; Nov 19. [Epub ahead of print]

39. Squizzato A, Romualdi E, Ageno W. Why should statins prevent venous thromboembolism? A systematic literature search and a call for action. J Thromb Haemost. 2006;4(9):1925-1927.

40. Agnelli G, Becattini C. Venous thromboembolism and atherosclerosis: common denominators or different diseases? J Thromb Haemost. 2006;4(9):1886-1890. 